\title{
A Study on the Interrelationship between Renal Calculi, Hormonal Abnormalities and Urinary Tract Infections in Iraqi Patients
}

\author{
Mohemid M. Al-Jebouri", Nadia Atalah \\ Department of Microbiology, College of Medicine, University of Tikrit, Tikrit, Iraq \\ Email: "profaljebouri@yahoo.com
}

Received December 7, 2011; revised January 18, 2012; accepted January 30, 2012

\begin{abstract}
Objective: The main objective of the present study is to assess the interrelationship between renal calculi, selected ions, thyroid and parathyroid hormones and urinary tract infections. Methods: This study was carried out on 150 patients attended Tikrit Teaching Hospital from 2008 to 2009. Stones and serum ions were analyzed utilizing relevant Biolab Company kits (France). Thyroid hormones were determined using ELISA microwells kit (Accuubind, USA) Parathyroid hormone was estimated by active I-PTH ELISA (DSL, USA). Urine cultures were done utilizing cystine-lactoseelectrolyte deficient (CLED) medium. Various isolated pathogens were conventionally identified. Results: Urine cultures revealed that $42 \%$ of the patients had urinary tract infections particularly with Enterobacteriaceae. $68 \%$ of the stones tested were calcium oxalate. Infective and noninfective stones were classified. Calcium ion was more elevated in patients examined. Hypothyroidism was prevalent but parathyroid hormone (PTH) was elevated among 14 patients only. Conclusions: The frequency of UTI was higher among urolithiasis patients and the common causative agents were Gram negative bacteria. Renal stones of calcium origin were predominant. Hormonal abnormalities were seen. 15 patients with hyperthyroidism revealed hypercalcemia and hypercalciuria.
\end{abstract}

Keywords: Urinary Tract Infection; Renal Stone; Hormones; Ions; Iraq

\section{Introduction}

Urolithiasis is a major health problem with its high morbidity, high cost management and potential for end stage renal disease [1]. Urinary calculi are the third most common affliction of the urinary tract, exceeded only by urinary tract infection (UTI) and pathogenic conditions of the prostate [2]. UTI is the most common bacterial infection across all age groups, and affects around $20 \%$ of women at some time during their lifetime. UTI is the second most clinical indication for antibiotic treatment in both primary and secondary care. It is also the most common hospital acquired infection, e.g. in the UK accounting for $23 \%$ of all infections [3]. UTI represents the commonest genitourinary disease in children and is the second commonest infection which affects them [4]. Infections of the urinary tract may present in a wide variety of ways. This ranges from asymptomatic bacteriuria to sepsis with multiorgans dysfunctions. Infections may arise from any part of the urinary tract such as the kidney as pyelonephritis, renal abscesses; the bladder as cystitis; the prostate as prostitis and prostate abscesses or the

${ }^{*}$ Corresponding author. urethra as urithritis [5]. A better understanding of the pathogenesis of UTI and the role of host and bacterial factors have improved the ability to identify the patients at risk and prevent or minimize sequelae. New diagnostic tests and antimicrobial agents that achieve high urinary levels can be administered orally and that are not nephrotoxic have significantly reduced the need for hospitalization for severe infections. Shorter course therapy and prophylactic antimicrobials have reduced the morbidity and cost associated with recurrent UTI.

Renal calculi are crystalline minerals deposits that form in the kidney. They develop from microscopic crystals in the loop of Henle, the distal tubule, or the collecting duct, and can enlarge to form visible fragments. Renal stone formation is the end results of a physiochemical process that involves nucleation of crystals from a supersaturated solution. The factors that influence crystals generation are urine volume, concentration of stone constituents, urinary $\mathrm{pH}$, the presence of nidus, and the balance among various physiochemical factors that inhibit or promote stone formation [4].

Stone results from a phase change in which dissolved salts condense into solids and all phase changes are 
driven by supersaturation (SS) which is usually approximated for such salt by the ratio of their concentration in urine to their solubilities. At SS less than one crystal of substance will dissolve and at SS values greater than one crystal can form and grow [6].

Hyperthyroid can cause renal stone. Hyperthyroid increases bone turn over resulting in hypercalcemia as many as $20 \%$ of hyperthyroid patients have high normal or mildly elevated serum calcium concentration. Hyperthyroid results in hypercalcemia which can lead to hypercalcuria, Hyperthyroidism also can lead to distal renal tubular acidosis (DRTA) which is considered an important cause of renal stone [7].

The parathyroid hormone (PTH) is the most important endocrine regulator of calcium and phosphorous concentration in the extracellular fluid [8]. It is secreted by parathyroid glands. Overactive parathyroid gland (parathyroidism) causes kidney stones in about $1 \%$ of Ca-stones patients. Renal stones are usually either $\mathrm{Ca}$-oxalate or $\mathrm{CaPO}_{4}$. Hyperparathyroid leads to hypercalcemia, hypercalcuria and it increases phosphate excretion in the urine (hyperphosphaturia) [9]. In a study carried out by Suh et al., 2006, found that four-fold increased prevalence of asymptomatic renal stone disease in patients with primary hyperparathyroidism compared to subjects not affected by this disorder [10]. Hyperparathyroidism can cause nephrolithiasis that may lead to urinary tract obstruction, infection and loss of renal function.

This type of work is not locally common, so we have carried out a series of attempts concerning the UTI and its predisposing factors particularly the intrinsic types as well as environmental parameters related to the subject as the UTI elevated in the last years in Iraq. The aim of the present study is an attempt to explore the interrelationship between blood levels of selected relevant hormones, ions, renal calculi and the co-incidence with urinary tract infections.

\section{Materials and Methods}

\subsection{Patients}

This study was conducted on the patients of Urology Department wards and/or from out patients consultation clinics in both Tikrit Teaching Hospital and Salahldeen Hospital of Tikrit city. The number of patients was 150 and they were recruited from October 2008 to August 2009. All the patients were having urolithiasis including 102 males and 48 females. One hundred and twenty stones were collected and submitted to clinical laboratory for chemical analyses. Blood samples were collected from patients with renal stones and submitted for analysis and estimation of T3, T4, TSH and PTH. Twenty control subjects were also involved to evaluate their serum hormone levels for comparison. Serum calcium, urine calcium, urine phosphate and urine specimens were also collected from all patients under study for bacteriological study.

\subsection{Urine Culture}

Cystine-lactose electrolyte deficient medium (CLED) was used for isolation of pathogens causing urinary tract infections according to manufacturer's instructions. The prepared media used for isolation, counting, identification and susceptibility testing were carried out after being solidified and dried $[11,12]$.

\subsection{Identification of Bacteria}

Bacterial species were conventionally identified [3]. API systems (bioMerieeux, France) was also utilized according to manufacturer's instructions.

\subsection{Chemical Analysis of Stones}

The obtained renal stones were analysed utilyzing kits from BIOLAB (France) according to manufacturer's instructions.

\subsection{Determination of Calcium and Phosphorous Ions}

These ions were determined using BIOLAB kits (France) according to manufacturer's instructions.

\subsection{Determination of Thyroid Hormones}

T3, T4 and TSH were determined utilizing ELISA Microwells kit from Accubind (USA) according to manufacturer's instructions.

\subsection{Determination of Parathyroid Hormone (PTH)}

PTH concentration in patients sera was determined using ACTIVE I-PTH ELISA kit of DSL (USA) according to manufacturer's instructions.

\subsection{Statistical Analyses}

Data collected were analysed by using Microsoft Office Excel 2007, Mann-Whitney test and F-test [13].

\section{Results}

Urine culture was done for all the 150 patients who were included in the present study. Only 63 patients had positive urine culture and 39 of them were females (Table 1).

The most common pathogen was Escherichia coli which was isolated from $22(32.8 \%)$ patients. Proteus mirabilis was also frequently isolated and its isolation frequency was almost $18 \%$. Almost $15 \%$ of the patients 
Table 1. The frequency of isolated bacteria from urine cultures of the patients studied.

\begin{tabular}{cc}
\hline Bacterial types & $\%$ \\
\hline Escherichia coli & 32.8 \\
Proteus mirabilis & 17.9 \\
Pseudomonas aeruginosa & 14.9 \\
Staphylococcus aureus & 8.9 \\
Klebsiella pneumoniae & 7.4 \\
Enterobacter aerogenes & 5.9 \\
Staphylococcus epidermis & 4.4 \\
Enterococcus faecalis & 4.4 \\
Serratia marcescens & 1.5 \\
Citrobacter freundii & 1.5 \\
\hline
\end{tabular}

were infected with Pseudomonas aeruginosa. The other bacteria were occasionally isolated. Four patients revealed mixed growth of the bacterial pathogens listed in the Table 1. Statistically, there was highly significant difference between male and females with UTI according to the distribution of the isolated bacteria ( $\mathrm{p}$-value less than $0.05, \mathrm{~W}=5133$ ).

Table 2 shows positive urine culture among patients who underwent lithiotomy or passed their stones spontaneously. Sixty three $(42 \%)$ of the patients had positive urine culture and the ratio of females to males was almost 1.6:1. The number of patients with infective stones who had positive urine culture was $8(5.3 \%)$ and $5(3.3 \%)$ of them were males. The number of patients with noninfective stone who had positive urine culture was $44(36 \%)$ and $28(23 \%)$ of them were females. Statistically, there was no significant difference in distribution of positive urine culture between the infectious stone and the noninfectious types according to sex using Mann-Whitney (W) test and Chi-square test (p-value more than 0.05).
In the present study, 120 stones were collected and analyzed (Table 3). The most predominant renal calculi were calcium oxalate and calcium oxalate urate types and their frequency was collectively almost $68 \%$. The most predominant cation was calcium $(92 \%)$ and the most predominant anion were oxalate, urate and phosphate and their percentages were $72.5,42.5$ and 22.5 respectively.

Table 3 shows that males were under risk of having renal stone more than females particularly with respect to hypothyroidism as $40 \%$ of the males were suffering from renal calculi. Calcium oxalate was predominant as well among males with hypothyroidism (17.5\%). Lower incidence of renal stone was seen among patients with hyperthyroidism $(5.8 \%)$. Calcium phosphate was the predominant type of renal calculi among patients with elevated parathyroid hormone (hyperparathyroidism) particularly males. Individuals with normal hormonal assays concerned showed almost similar incidence of stones formation with average did not exceed $18 \%$.

Urine calcium was highly elevated among patients studied (Table 4). Thirty three (22\%) of the patients examined showed an elevation in serum calcium particularly among patients with hyperthyroidism followed by the patients suffered from an elevation in parathyroid hormone, i.e. $8 \%$ and $6.7 \%$ respectively. The highest estimated value of urine phosphate was recorded among males with hyperparathyroidism $(6.7 \%)$. In general, males revealed higher serum and urine ions compared to females. Statistically, there was no significant difference

Table 2. The relationship between urinary tract infections and stone types with reference to sex of patients.

\begin{tabular}{cccc}
\hline \multirow{2}{*}{ Stone type } & \multicolumn{2}{c}{ Sex } & \multirow{2}{*}{ Total } \\
\cline { 2 - 3 } & Males & Females & \\
\hline Infectious stone & $5(4 \%)$ & $3(2.5 \%)$ & $8(40 \%)$ \\
Noninfectious stone & $16(13 \%)$ & $28(23 \%)$ & $44(36.7 \%)$ \\
Total & $21(17 \%)$ & $31(26 \%)$ & $52(43 \%)$ \\
\hline
\end{tabular}

Table 3. The relationship between selected hormonal abnormalities and stones chemical composition.

\begin{tabular}{cccccccccc}
\hline \multirow{2}{*}{ Types of Stones } & \multirow{2}{*}{ Number (\%) } & \multicolumn{2}{c}{ Hyperthyroid } & \multicolumn{2}{c}{ Hypothyroid } & \multicolumn{2}{c}{ Hyperparathyroid } & \multicolumn{2}{c}{ Normal Hormones Levels } \\
\cline { 3 - 9 } & & $\mathrm{F}$ & $\mathrm{M}$ & $\mathrm{F}$ & $\mathrm{M}$ & $\mathrm{F}$ & $\mathrm{M}$ & $\mathrm{F}$ & $\mathrm{M}$ \\
\hline $\mathrm{CaPO}_{4}$ & $12(10 \%)$ & 0 & 1 & 0 & 0 & 2 & 6 & 2 & 1 \\
$\mathrm{CaMgPh}$ & $12(12 \%)$ & 0 & 0 & 0 & 6 & 0 & 0 & 3 & 3 \\
$\mathrm{U} . \mathrm{A}$. & $9(7.5 \%)$ & 0 & 0 & 6 & 3 & 0 & 0 & 0 & 0 \\
$\mathrm{U} . \mathrm{A} .+\mathrm{CaO}_{\mathrm{x}}$ & $42(35 \%)$ & 0 & 3 & 6 & 18 & 0 & 0 & 12 & 3 \\
$\mathrm{CaO}_{\mathrm{x}} \mathrm{Ph}$ & $3(2.5 \%)$ & 0 & 0 & 0 & 0 & 1 & 0 & 0 & 2 \\
$\mathrm{CaO}_{\mathrm{y}}$ & $42(35 \%)$ & 0 & 3 & 0 & 21 & 0 & 0 & 6 & 12 \\
$\mathrm{Total}$ & $120(100 \%)$ & 0 & 7 & 12 & 48 & 3 & 6 & 23 & 21 \\
\hline
\end{tabular}

$\mathrm{CaPo}_{4}$, calcium phosphate; $\mathrm{CaMgPh}$, calcium magnesium phosphate; U.A., uric acid; U.A. $+\mathrm{CaO}_{\mathrm{x}}$, uric acid and calcium oxalate; $\mathrm{CaO} \mathrm{Ph}_{\mathrm{x}}$, calcium oxalate phosphate; $\mathrm{CaO}_{\mathrm{x}}$, calcium oxalate. $\mathrm{F}$, female; $\mathrm{M}$, male. 
Table 4. The relationship between selected ions and hormonal abnormalities.

\begin{tabular}{|c|c|c|c|c|c|c|c|}
\hline \multirow[t]{2}{*}{ Ions } & \multirow[t]{2}{*}{$\begin{array}{l}\text { Patients } \\
\text { No. (\%) }\end{array}$} & \multicolumn{2}{|c|}{ Hyper PTH } & \multicolumn{2}{|c|}{ Hyperthyroidism } & \multicolumn{2}{|c|}{ Hypothyroidism } \\
\hline & & $\mathrm{F}^{*}$ & M & $\mathrm{F}$ & M & $\mathrm{F}$ & M \\
\hline S.Ca & $33(22 \%) 4$ & $(2.7 \%)$ & $10(6.7 \%)$ & $3(2 \%)$ & $12(8 \%)$ & $3(2 \%)$ & $0(0 \%)$ \\
\hline U.Ca & $99(66 \%) 4$ & $(2.7 \%)$ & $10(6.7 \%)$ & $3(2 \%)$ & $12(8 \%)$ & $9(6 \%)$ & $24(16 \%)$ \\
\hline U.Ph & $39(26 \%) 4$ & $(2.7 \%)$ & $10(6.7 \%)$ & $0(0 \%)$ & $6(4 \%)$ & $6(4 \%)$ & $9(6 \%)$ \\
\hline
\end{tabular}

${ }^{*}$ F, female; M, male. PTH, parathyroid hormone; S.Ca, serum calcium; U.Ca, urine calcium; U.Ph, urine phosphate.

between males and females according to serum calcium, urine calcium and urine phosphate $(\mathrm{P}$ value more than $0.05)$.

Table 5 shows the interrelationship between selected hormonal abnormalities (T3, T4, TSH and PTH) and UTI. It was quite clear that urinary tract infection was more prevalent among males with relation to abnormalities in thyroid and parathyroid hormones particularly among patients with hypothyroidism and $54(78 \%)$ of the patients studied were interrelated with this disease. Statistically, there was a significant difference between males and females according to T3 (p-value less than 0.05).

\section{Discussion}

The most organisms causing UTI isolated in the present study were Gram negative bacteria dominated by Enterobacteriaceae (Table 1) particularly Escherichia coli. In a study carried out in Iraq, Al-Jobouri [14] almost showed the same distribution of bacteria concerning UTI. Morever, Navaneeth $[15,16]$ also concluded nearly similar results elswhere. Furthermore, noninfction stones were more prevalent among the patients studied. Statistically, there was no significant difference in distribution of positive urine culture between infection and noninfection stones (p-value more than 0.1). The same conlusion was seen by other workers elsewhere $[15,16]$. Proteus mirabilis and Klebsiella pneumoniae were seen to be associated with phosphate stones whereas E. coli commonly related to Ca-oxalate stones and this was might be related to $\mathrm{pH}$ of urine $[17,18]$.

In the present study, almost $53 \%$ of the patients had pure stones and the remaining subjects were suffered from mixed stones. This result was similar to that concluded by other workers [19]. In contrast, Al-Jobouri [14] found that the mixed stones were the most prevalent types. The most predominant constituent of both mixed and pure stones was Ca-oxalate $(72.5 \%)$. The present result revealed similar conclusion of Dayani et al. [17], Bulo et al. [19] and Al-Jebouri [14]. The females were more prone to develop phosphate stones and males were under risk of having Ca-oxalate and uric acid stones. The males usually more affected by calcic and uric stones, might be due to risk factors like hypercalcuria and hyperuricuria and they might crystilized. The prevalence of $\mathrm{Ca}$-oxalate, Ca-phosphate and $\mathrm{Ca}$-oxalate-urate stones varied depending on environmental factors, especially dietry intake and life style, whereas infection stones are less influenced by these conditions [19].

The parathyroid hormonal assays using ELISA technology showed that 14 ptients $(9 \%)$ had abnormal values i.e. more than $45.5 \mathrm{pg}$. One male patient had both hyperthyroidism and elevated PTH. These hormonal assays were almost similar to those concluded by Yendet [19] but they were in contrast with those of Fuss et al. [20]. However, overactive parathyroid gland causes kidney stones particularly Ca-oxalate or Ca-phosphate [21]. The patients with hyperthyroidism revealed an elevated serum and urine calcium. Hyperthyroidism causes excessive loss of calcium in urine than that caused by hyperparathyroidism [22]. Moreover, hypothyroidism was mentioned as a disease that correlates with formation of urolithiasis. Hypothyroidism decreases urine volume by decreasing urine output and decreasing renal water excretion by decreasing glomerular filteration rate. Hyperthyroidism also cause acquired renal tubular acidosis. This disease might cause neurogenic bladder as a result of metabolic disturbances. Urine acidification might also caused by hypothyroidism [22]. This disease tends to be associated with diminished resistance to infection [23]. However, Iraqi citizens usually under effect of high environmental extreme temperature that exceed $50^{\circ} \mathrm{C}$ in the summer leading to dehydration, high concentration of serum ions and stress affecting their hormones and metabolism.

\section{Conclusion}

The frequency of UTI among urolithiasis patients was

Table 5. The relationship between selected hormonal abnormalities and urinary tract infections.

\begin{tabular}{|c|c|c|c|c|c|c|}
\hline \multirow{2}{*}{ Hormonal abnormalities } & \multicolumn{2}{|c|}{ UTI } & \multicolumn{2}{|c|}{ No UTI } & \multicolumn{2}{|c|}{ Total } \\
\hline & Female & Male & Female & Male & Female & Male \\
\hline Hypothyroidism & $11(16 \%)$ & $13(18.8 \%)$ & $4(5.7 \%)$ & $41(59.5 \%)$ & $15(22 \%)$ & $54(78 \%)$ \\
\hline Hyperthyroidism & $2(13.3 \%)$ & $2(13.3 \%)$ & $1(6.7 \%)$ & $10(66.7 \%)$ & $3(20 \%)$ & $12(80 \%)$ \\
\hline PTH above the average of The control (45.5 pg) & $3(22 \%)$ & $2(14 \%)$ & $1(7 \%)$ & $8(57 \%)$ & $4(28 \%)$ & $10(72 \%)$ \\
\hline
\end{tabular}

UTI, urinary tract infection; PTH, parathyroid hormone. N.B., some patients had more than one metabolic abnormalities. 
$42 \%$ which was greater than that of non-urolithiasis patients. UTI was more predominant among females. Escherichia coli was the predominant pathogen associated mostly with non infection stone. Urolithiasis was more frequent among males. Ca-oxalte stones were predominant. Sixty four percentage of the patients were with hormonal abnormalities concerning parathyroid and thyroid glands. Serum and urine calcium was related to hyperthyroidism. Patients with elevated parathyroid hormone had hypercalcuria, hypercalcemia and hyperphosphaturia.

\section{REFERENCES}

[1] K. El-Shaid, H. Mughal and K. Kapoor, "Epidemiological Profile Mineral Metabolic Pattern and Crystallographic Analysis of Urolithiasis in Kuwait," European Journal of Epidemiology, Vol. 13, No. 2, 1997, pp. 229-234. doi:10.1023/A:1007346727944

[2] L. Marshal and M. D. Stollre, "Urinary Stone Disease," In: E. D. Tanagho and J. W. Aninch, Eds., Smith's General Urology, Lange Medical Books/McGraw-Hill Medical Publishing Division, New York, 2004, pp. 268-271.

[3] M. M. Al-Jebouri and H. Al-Dobony, "Incidence of Asymptomatic Bacteriuria among Secondary School Girls in Mosul, Iraq," Iraqi Medical Journal, Vol. 8, No. 2, 1985, pp. 112-117.

[4] T. Al-Momani, "Microbiological Study of Urinary Tract Infection in Children at Princess Haya Hospital in South Jordan," Middle East Journal of Family Medicine, Vol. 14, No. 2, 2006, pp. 15-18.

[5] N. Sen, A. Matthias and J. P. Raj, "Role of Critical Care in Urological Sepsis," Indian Journal of Urology, Vol. 22, No. 2, 2006, pp. 105-112. doi:10.4103/0970-1591.26562

[6] M. M. Al-Jebouri, "Medical Bacteriology," Mosul University Press, Mosul, 1990.

[7] M. S. Parmer, "Kidney Stones," British Medical Journal, Vol. 328, No. 4, 2004, pp. 1420-1424. doi:10.1136/bmj.328.7453.1420

[8] N. Ciftcioglu, M. Bjorklund, K. Kuorikoski, et al., "Nanobacter: An Infectious Cause for Kidney Stone Formation," Kidney International, Vol. 56, No. 5, 1999, pp. 1893-1898. doi:10.1046/j.1523-1755.1999.00755.x

[9] M. T. Harrison, R. M. Harden and W. D. Alexander, "Some Effects of Parathyroid Hormone in Thyrotoxicosis," Journal of Clinical Endocrinology and Metabolism, Vol. 24, No. 2, 1964, pp. 214-217. doi:10.1210/jcem-24-2-214

[10] J. M. Suh, J. J. Corona and J. M. Monchik, "Primary Hyperparathyroidism: Is There an Increased Prevalence of
Renal Stone Disease?" American Journal of Roentgenology, Vol. 191, No. 3, 2008, pp. 908-911. doi:10.2214/AJR.07.3160

[11] W. G. Robertson, S. Germani and G. Vespasiani, "Stones and Urinary Tract Infections," Urolology and Infection, Vol. 79, Suppl. 1, 2007, pp. 32-36.

[12] S. T. Cowan, "Cown and Steel's Manual for Identification of Medical Bacteria," Cambridge University Press, Cambridge, 1976.

[13] N. J. Castylan, "Nonparametric Statistics," McGrowthat, New York, 1989.

[14] O. A. H. Al-Jebouri, "The Relationship between Urinary Calculi Types and Urinary Tract Infections among Patients in Tikrit District," M.Sc. Thesis, University of Tikrit, Tikrit, 2006.

[15] M. M. Al-Jebouri, "The Effect of Sublethal Concentrations of Disinfectants on Antibiotic-Resistant Staphylococcus aureus," Journal of Hospital Infection, Vol. 14, No. 4, 1989, pp. 14-19.

[16] A. J. Howard, J. T. Magee, K. A. Fitzgerald, et al., "Factors Associated with Antibiotics Resistance in Coliforms from Community UTI in Wales," Journal of Antimicrobial Chemotherapy, Vol. 47, No. 3, 2001, pp. 305-313. doi:10.1093/jac/47.3.305

[17] M. Farooq, M. C. H. Anwaar, M. Albukhari, et al., "Urinary Calculi; Biochemical Profile Stones Removed from Urinary Tract," Professional Medical Journal, Vol. 14, 2007, pp. 6-10.

[18] P. B. Cooke, J. R. Nassim and J. Collins, "The Effect of Thyrotoxicosis upon the Metabolism of Calcium, Phosphorous and Nitrogen," Quarterly Journal of Medicine, Vol. 28, No. 4, 1959, p. 505.

[19] E. R. Yendt, "Renal Calculi," Canadian Medical Journal, Vol. 102, No. 19, 1970, pp. 479-489.

[20] M. Fuss, T. Pepersack, J. Corvilain, et al., "Infrequency of Primary Hyperparathyroidism in Renal Stone Formers," British Journal of Urology, Vol. 62, No. 1, 1988, p. 4. doi:10.1111/j.1464-410X.1988.tb04254.X

[21] R. P. Holmes, H. O. Goodman and D. G. Assimos, "Contribution of Dietary Oxalate to Urinary Oxalate Excretion," Kidney International, Vol. 59, No. 1, 2001, pp. 270 276. doi:10.1046/j.1523-1755.2001.00488.x

[22] S. Corbetta, A. Baccarelli, A. Aroldi, et al., "Risk Factors Associated to Kidney Stones in Primary Hyperparathyroidism," Journal of Endocrinology Investigation, Vol. 28, No. 2, 2005, pp. 122-128.

[23] L. Hall-Stoodley, J. W. Costerton and P. Stoodley, "Bacterial Biofilms: From Natural Environment to Infectious Disease," Nature Reviews Microbiology, Vol. 2, No. 2, 2004, pp. 95-108. doi:10.1038/nrmicro821 